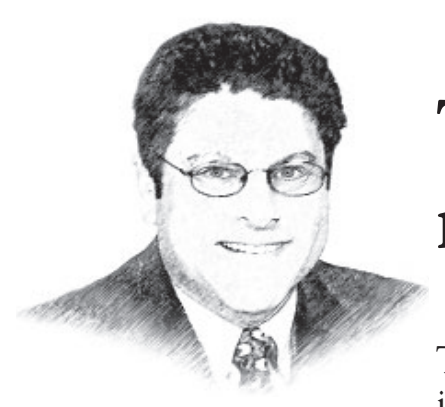

\title{
The initial viral infection may be only part of the story
}

The concept of "long COVID" is now entrenched in our vocabulary and in the minds of our patients, but the pathogenesis of this protracted syndrome is thus far incompletely understood. Are there pockets of slowly dividing virus or incompletely cleared viral remnants driving a low-level inflammatory response? Is there a central nervous system reservoir of infected neurons contributing to an intense sense of fatigue, brain fog, and sometimes mood changes? Is there persistent infection of vascular cells impacting vascular regulation of blood flow to selected organs?

While we cannot answer these questions now, we clearly see that the coronavirus elicits pathologic syndromes far afield from the respiratory tissues that the virus primarily targets for infection.

A virus eliciting effects seemingly distinct from its primary infection is not a new concept at all, of course. Epstein-Barr virus is recognized as causing not only a lasting malaise in some patients, but also a reactivation syndrome and lymphoproliferative disorders. Hepatitis C and B virus infections are linked to the development of delayed hepatocellular carcinoma in the setting of hepatic fibrosis, as well as several systemic vasculitic disorders occurring during active infection. Parvovirus is associated with aplastic anemia and human papillomavirus is strongly linked to various carcinomas.

But the most common viral infection associated with delayed clinical events is the double-stranded DNA varicella-zoster alpha-herpesvirus (VZV), the causative agent for "chicken pox" and shingles. Seminal work from the laboratories of the late Dr. Don Gilden and others has demonstrated that the viral DNA exists in neurons, in a nonintegrated form. Reactivation is best recognized by the appearance of pruritic, painful vesicles in an asymmetric dermatomal distribution. While often no definite trigger for reactivation with viral replication is recognized, it is felt that loss of cellular immunity is at least permissive, and this is increasingly recognized in a minority of patients treated with JAK inhibitors for rheumatoid arthritis and other inflammatory disorders. How a decrease in cellular immunity affects intracellular viral replication is not entirely clear, but more readily understood is that patients who are immunosuppressed often have a more difficult time containing and controlling the reactivation when it occurs. It is often said that stress may also bring about reactivation, and several fascinating studies have demonstrated that after space travel, astronauts experience a self-limited asymptomatic reactivation of VZV as detected by the presence of salivary viral DNA. ${ }^{1}$

Postherpetic neuralgia is the best-known complication of dermatomal reactivation, and although some risk factors are known, we cannot predict who will develop it, nor do we know how to prevent it. The virus has been detected in cerebral blood vessels, and a VZV vasculopathy, often with giant cells and adventitial inflammation, has been associated with stroke syndromes and, controversially, with giant cell arteritis. VZV stroke syndromes may occur weeks or months after an episode of dermatomal zoster. VZV DNA or, more commonly, anti-varicella IgG antibody can be found in the cerebrospinal fluid of affected patients.

A myelopathy or segmental peripheral episode of motor weakness can follow an

doi:10.3949/ccjm.88b.09021 
episode of typical zoster. Segmental weakness generally occurs in the area of the zoster outbreak, but not always. Myelopathy may affect legs and sphincter tone and has also been rarely described after infection with cytomegalovirus, another herpesvirus.

Since pain and neurologic complications before, during, or after VZV infection can occur in the absence of the classic skin findings (zoster sine herpete), ${ }^{2}$ it is important to recognize the wide spectrum of regional VZV syndromes that can occur. How many times have I not recognized impending zoster in the setting of radiculopathy or an unusual regional pain syndrome? I know that I have on multiple occasions diagnosed prezoster rash-pain syndromes - oddly, it seems, more in family and friends than in patients in my office. (Patients these days probably cannot get an appointment in time before the vesicles appear.)

It is with this background in mind that I invite you to read the Clinical Picture in this issue by Dr. Mizumoto (page 480). ${ }^{3}$

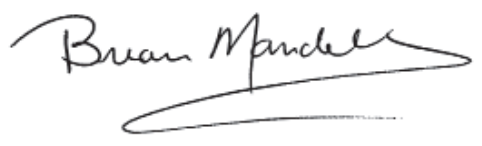

Brian F. Mandell, MD, PhD

Editor in Chief

1. Mehta SK, Laudenslager ML, Stowe RP, Crucian BE, Sams CF, Pierson DL. Multiple latent viruses reactivate in astronauts during Space Shuttle missions. Brain Behav Immun 2014; 41:210-217. doi:10.1016/j.bbi.2014.05.014

2. Gilden DH, Wright RR, Schneck SA, Gwaltney JM Jr, Mahalingam R. Zoster sine herpete, a clinical variant. Ann Neurol 1994; 35(5):530-533. doi:10.1002/ana.410350505

3. Mizumoto J. Abdominal pseudohernia due to herpes zoster. Cleve Clin J Med 2021; 88:480-481. doi:10.3949/ccjm.88a.20178 